ARTICLE

Received 18 Sep 2014 | Accepted 10 Feb 2015 | Published 17 Mar $2015 \quad$ DOl: 10.1038/ncomms7590

\title{
Dynamically self-assembled silver nanoparticles as a thermally tunable metamaterial
}

\author{
Wiktor Lewandowski ${ }^{1}$, Martin Fruhnert ${ }^{2}$, Józef Mieczkowski ${ }^{1}$, Carsten Rockstuhl $^{3,4} \&$ Ewa Górecka ${ }^{1}$
}

The availability of metamaterials with properties that can be actively tuned is crucial for the future development of various metamaterial-based technologies. Here we show that by using silver nanoparticles equipped with a thermally responsive organic coating a metamaterial is obtained with reversibly switchable properties. The material investigated exhibits dynamic self-assembly resulting from temperature-dependent changes of organic coating shape, which translates to a switchable spatial distribution of the silver nanoparticles. This in turn strongly influences the optical properties of the entire material. The measured optical characteristics of the material are in excellent agreement with theoretical calculations, which allow us to use the latter to predict a dynamically tunable epsilon-near-zero behaviour of the metamaterial. The suggested methodology opens new routes for tunable metamaterials that operate in the visible region and will enable various applications for soft-matter-based optical devices.

\footnotetext{
${ }^{1}$ Faculty of Chemistry, University of Warsaw, 1 Pasteura street, 02-093 Warsaw, Poland. ${ }^{2}$ Institute of Condensed Matter Theory and Solid State Optics, Abbe Center of Photonics, Friedrich-Schiller-Universität Jena, 07743 Jena, Germany. ${ }^{3}$ Institute of Theoretical Solid State Physics, Karlsruhe Institute of Technology, 76131 Karlsruhe, Germany. ${ }^{4}$ Institute of Nanotechnology, Karlsruhe Institute of Technology, 76021 Karlsruhe, Germany. Correspondence and requests for materials should be addressed to W.L. (email: wlewandowski@chem.uw.edu.pl) or to E.G. (email: gorecka@chem.uw.edu.pl).
} 
S elf-assembly (SA) of nanoparticles (NPs) into ordered structures is a formidable challenge in many branches of material science, especially for metamaterials research ${ }^{1-4}$. Beyond being fascinating from an intellectual perspective, selfassembled metamaterials provide a clear solution to some open problems that have to be solved before metamaterials can evolve to a mature technology. This especially concerns the availability of metamaterials in large-scale quantities and as bulk materials. To date, SA of NPs provided access to materials exhibiting topological darkness ${ }^{5}$, epsilon-near-zero (ENZ) behaviour $^{6-8}$, magnetic-based Fano resonance scattering at optical frequencies ${ }^{9}$, efficient absorbance properties ${ }^{10}$ and also enabled optical cloaking ${ }^{11,12}$. However, the established SA approaches to form metamaterials result in static structures. This severely limits their functionality since metamaterials with tunable characteristics are often required for many budding applications $^{13-21}$.

Various approaches have been explored to achieve dynamically reconfigurable metamaterials, including embedded varactors ${ }^{22}$, metamaterial hybridization with liquid crystals $^{21,23}$, the use of electromechanical $^{20}$, elasto-electromagnetic ${ }^{17}$ and laserinduced $^{18}$ modulation as well as exploitation of phase-change materials ${ }^{14-16}$. However, dimensions of these structures are usually on the order of $\mu \mathrm{m}$ or hundreds of $\mathrm{nm}$, which results in operation wavelengths outside the visible region, highlighting the need for tunable metamaterials made of nanocrystal solids.

To this end, broad tunability of nanocrystal solids' properties has been achieved, for example, by marked decreasing interparticle spacing, which enhances interparticle coupling. Potentially the best developed strategy of this kind is based on exchange of large, organic ligands that are usually used in the nanocrystals synthetic process to small inorganic molecules ${ }^{24-27}$. Some conspicuous examples of application of this approach include switching insulating $\mathrm{PbSe}$ nanocrystal arrays to an n-type semiconductor $^{28}$ and dielectric-to-metal transition of Au NPs aggregates $^{2}$. However, these changes are predominantly irreversible, whereas active tunability of properties is necessary to achieve tunable metamaterials.

Those limitations may be overcome by using dynamic SA (DSA) of NPs. DSA would unlock the opportunities of an active engineering of aggregate structures and associated properties as lately evidenced by theoretical modelling 29,30 . Recently, a handful of DSA methods have been reported ${ }^{31-34}$, but most of these techniques rely unfortunately on solvated environments, which result in very low NP-filling fractions, or are limited to twodimensional systems ${ }^{35,36}$. Therefore, the application of these methods in metamaterials research is limited. It would be of utmost importance to obtain large metal-filling fractions to access materials that deviate from weakly dispersive dielectric properties exhibited by strongly diluted metallic NP arrays. Devising new DSA strategies to obtain reconfigurable, densely packed NP assemblies is therefore of paramount importance. Notably, DSA of metal particles is a complementary approach to the use of static SA of nanocrystals made of phase-change material, which was recently reported ${ }^{13}$. Both provide access to reversible, thermally switchable metamaterials and underline the surge for tunable metamaterials based on NPs.

Here, we show that DSA of NPs can lead to a system with theoretically predicted tunable metamaterial properties. To achieve this we used a SA strategy based on liquid-crystalline (LC) surface ligands. Recently, this approach has been intensively explored for both non-plasmonic and plasmonic $\mathrm{NPs}^{37-45}$. Also, this strategy was shown to enable the formation of dynamic assemblies ${ }^{46,47}$. For example, Wojcik et al. ${ }^{47}$ prepared a gold NP aggregate, which exhibited structural polymorphism-lamellar, rectangular, columnar and hexagonal phases were identified for a single sample at various temperatures. A LC strategy to DSA was also used by Umadevi et al. ${ }^{46}$ to create magneto-responsive materials made of LC-coated gold nanorods, in which spatial orientation of the rods was changed with a handheld magnet. However, in none of these systems were optical properties of the assemblies investigated. Another strategy for achieving DSA of NPs into dense arrays is using polymer nanocomposites ${ }^{48-50}$. Unfortunately, this strategy suffers from the complexity of achieving initial dispersion of NPs, low NP loadings ${ }^{51}$ and usually collective interactions of NPs were not examined. Only lately, chiral plasmonic DNA nanostructures with switchable circular dichroism ${ }^{52}$, and switchable dispersion of $\mathrm{Au}$ NPs with reversible tunability of the aggregate optical characteristic were obtained ${ }^{50}$. Plasmonic band shifting by $5 \mathrm{~nm}$ was observed in the latter report after thermal annealing of the material for tens of minutes. However, from the applicative point of view it would be advisable to improve two parameters of such systems-enhance kinetics of the optical tunability and broaden the range of the optical response $e^{53}$.

Herein, we report the synthesis of a dense Ag NP aggregate that undergoes heat-induced, reversible spatial reconfiguration from lamellar to the isotropic phase. Notably, this reorganization results in NP nearest-neighbour distance variation allowing active engineering of collective interactions between NPs. The relatively large separation between nanocrystals assured by organic coating does not allow us to observe marked changes of properties, for which achieving smaller interparticle gaps is necessary, therefore the expected tunability is limited to the strongly dispersive dielectric regime. The switching behaviour is evidenced by shifting of the aggregate plasmon band maxima position. We achieve tunability in the range of up to $20 \mathrm{~nm}$ for slow cooling and heating (tens of minutes). We also present the possibility of fast switching (ten of seconds) but with slightly lower optical response-14-nm shift. Importantly, the DSA methodology presented here allows continuous tunability of plasmonic properties, in contrast to most of DSA methods reported to date, which usually allow only switching between on and off states of the collective interactions. To fully confirm that the optical response of our material emerges from structural reorganization of NPs, we calculate extinction spectra of the aggregate obtained by slow cooling/heating using full-wave electromagnetic simulation tools, and found the modelled spectra to be in excellent agreement with the experiment. This numerical analysis also provides insight into metamaterial properties of the slowly switched system, indicating tunable ENZ behaviour. ENZ properties are further confirmed by ellipsometric measurements, which enable us to extract effective optical properties of the system. Lately, ENZ materials attract considerable interest, since they allow for precise phase manipulation of light ${ }^{54-56}$. ENZ materials based on nanocrystal solids that enable guiding and confining signals on the nanometre scale are especially interesting. Also, they can be designed to work in the entire visible spectrum ${ }^{57}$, which is indispensable for future optical fibre and optical computing technologies. In contrast to static examples of such materials ${ }^{6-8}$, here we report a NP-based material that exhibits theoretically predicted tunable ENZ properties enabling broadband function.

\section{Results}

Design and synthesis of hybrid NPs. To construct a tunable NP aggregate with likely metamaterial properties, we have synthesized LC-coated silver NPs following a two-step procedure shown in Fig. 1 (refs 21,35). First, ca. $4.7 \pm 0.5-\mathrm{nm}$ diameter (as determined with transmission electron microscopy (TEM), Fig. 1a) dodecanethiol-coated silver NPs were obtained using a previously reported ${ }^{38}$ modification of the Wang procedure ${ }^{58}$. Then, in a ligand-exchange reaction, a promesogenic molecule 


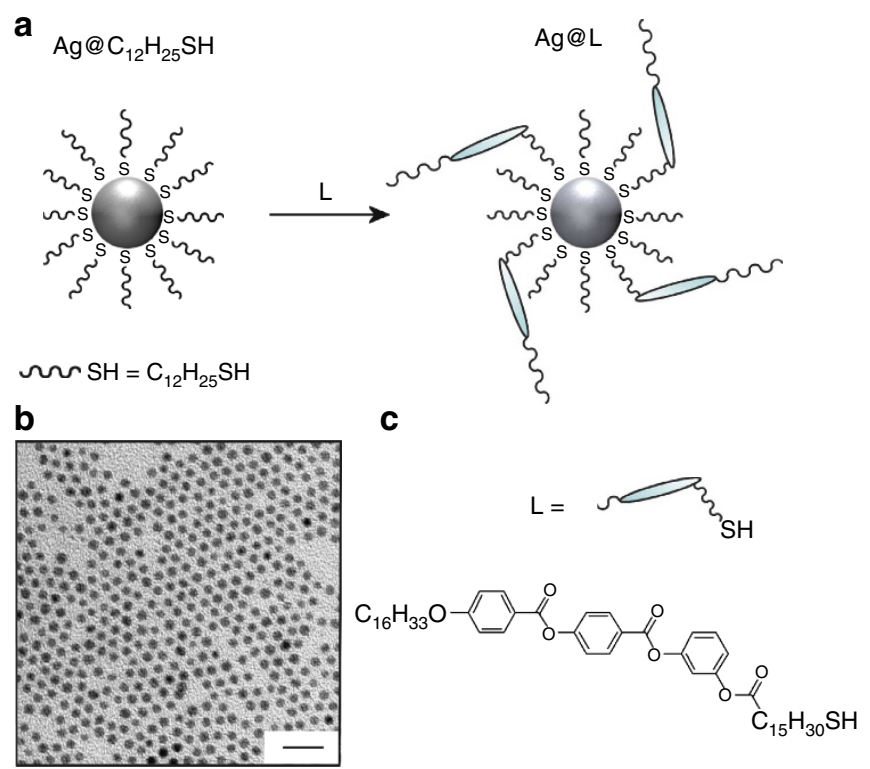

Figure 1 | Design and synthesis of hybrid Ag@L nanoparticles.

(a) Scheme ligand-exchange reaction on dodecanethiol-coated Ag NPs (Ag@ $\mathrm{C}_{12} \mathrm{H}_{25} \mathrm{SH}$ ) leading to the hybrid material Ag@L. (b) TEM image of $\mathrm{Ag} @ \mathrm{C}_{12} \mathrm{H}_{25} \mathrm{SH}$ nanoparticles, scale bar, $20 \mathrm{~nm}$. (c) Molecular structure of the promesogenic ligand $\mathrm{L}$.

(L, Fig. 1b, details of the synthesis are given in Methods, Supplementary Note 1 and Supplementary Fig. 1) was introduced into the NP surface, yielding hybrid silver NPs (Ag@L, Fig. 1c). The ligand-exchange reaction did not affect mean diameter of NPs but slightly increased metallic core size distribution $(4.7 \pm 0.6 \mathrm{~nm}$; as determined with TEM). It should be noticed that the free compound L does not form LC phases and melts directly into the isotropic phase at $\sim 87^{\circ} \mathrm{C}$, but mesogenic properties of the ligand are not prerequisite for inducing SA of NPs into the LC phase ${ }^{59,60}$. Also, molecules with similar architecture to $\mathrm{L}$, comprising three aromatic rings with ester linkages, were reported to support formation of LC phases ${ }^{61}$. Therefore, $\mathrm{L}$ can be viewed as a promesogenic structure in which a small change of substituents would result in LC properties. Terminal groups of the promesogenic molecule were chosen based on our previous experience with LC-NPs ${ }^{38,59}-$ hexadecyloxy chain was used to provide fluidity to the hybrid system, while a 15-carbon-length mercapto-functionalized alkyl chain assured flexibility of the ligand and served as a docking moiety to the NP surface. To evaluate the chemical nature of Ag@L NPs, X-ray photoelectron spectroscopy (XPS) and wideangle X-ray diffraction were used (see Supplementary Figs 2 and 3 , respectively). Wide-angle X-ray diffraction confirmed that metallic cores of NPs are composed of silver (cubic structure). XPS revealed the presence of expected atoms in the sample: silver, sulfur, carbon, oxygen and silicon (coming from the substrate) and allowed for quantitative elemental analysis (see details in Methods), confirming successful synthesis. The exact composition of the organic coating of A@L NPs was determined based on thermogravimetric analysis (TGA; see Supplementary Fig. 4) as described previously ${ }^{38}$. The organic shell of Ag@L is composed of ca. 240 alkane thiols and $260 \mathrm{~L}$ molecules, which in total is close to the number of alkanethiol ligands (520) grafted on the NP before the ligand-exchange reaction.

Switching material structure. To investigate SA properties of Ag@L NPs, we have used temperature-dependent small-angle
$\mathrm{X}$-ray diffraction (SAXRD) measurements and TEM imaging (Fig. 2). For SAXRD a small portion of the Ag@L solution was dropcasted onto kapton foil and heated. At elevated temperatures (above $100^{\circ} \mathrm{C}$ ) a single, diffuse signal corresponding to the mean interparticle distance of ca. $7.2 \mathrm{~nm}$ was observed (as measured at $120^{\circ} \mathrm{C}$, Fig. 2a), evidencing isotropic distribution of metallic cores within the sample-this structure will be referred to as the Iso phase. Subsequent lowering of the sample temperature resulted in a distinctive change of the diffraction pattern-three narrower Bragg scattering rings appeared, evidencing development of a long-range-ordered structure (Fig. 2b), resembling a LC lamellar structure. In this structure, metallic cores would form layers with well-developed interlayer distance of ca. $8.4 \mathrm{~nm}$ and interparticle (center-to-center) distance within the layer ca. $6.1 \mathrm{~nm}$ (as measured at $30^{\circ} \mathrm{C}$ ). For unambiguous determination of the structure we have attempted to prepare a monodomain sample by shearing the dropcasted Ag@L NPs at $50^{\circ} \mathrm{C}$. SAXRD measurements of the shear-aligned sample (see Supplementary Fig. 5) revealed two orthogonal signals indexed as (10) and (01), which are characteristic to a lamellar phase ${ }^{38,59,62}$ and confirm the initial structure assignment. On the basis of the broadness of the (01) signal, we assume that the metallic cores of NPs are arranged on a rectangular lattice within the layer, although hexagonal packing is another possibility. This low-temperature structure will be referred to as Lm. It should be noted that the $\mathrm{Lm}$ phase is no typical liquid-crystal, since it does not show optical birefringence. SAXRD measurements alone provide a proof of DSA properties of hybrid NPs, since reversible switching between the two states of the aggregate, $\mathrm{Lm}$ and Iso, was possible by varying the temperature between 30 and $120^{\circ} \mathrm{C}$. The kinetics of the process will be discussed later, when considering optical properties of the material.

To further confirm the above structural analysis we have turned to TEM imaging. Hybrid NPs were dropcasted onto a TEM grid that was subsequently heated to $120^{\circ} \mathrm{C}$ and cooled instantly by removing from a heating stage. Regions corresponding to both Iso (Fig. 2c) and Lm (Fig. 2d) arrangements of NP metallic cores were found with interparticle distances almost identical to those derived from SAXRD; for the Lm phase, the measured interlayer distance was $8.5 \pm 0.4 \mathrm{~nm}$, while for Iso the interparticle distance was $7.3 \pm 0.8 \mathrm{~nm}$.

By combining SAXRD and TEM results we were able to propose a model of the spatial arrangement of the Ag@L NPs cores (Fig. 2e,f) and consequently translate it into the organic corona shape. In the Iso phase, both promesogenic and alkyl ligands are evenly distributed in space forming a spherical entity, while in the Sm structure L molecules are mainly located in between the layers, and only partially contribute to the spacing within the layer. It is therefore the rearrangement of promesogenic ligands that causes the spatial rearrangement of NPs' metallic cores. This behaviour has been previously observed for smaller gold $\mathrm{NPs}^{59,62}$ and can be viewed as experimental realization of theoretically predicted DSA of shape-shifting particles ${ }^{63}$.

Switching material plasmonic properties. Figure 3 shows the dynamic optical characteristic of the Ag@L NP aggregate. Ultraviolet-visible absorption measurements (see details in Methods) revealed a 20-nm blue shift, from 466 to $446 \mathrm{~nm}$, of the plasmonic band $\left(\lambda_{\max }\right)$ when the LC-NP aggregate was heated from 30 to $120^{\circ} \mathrm{C}$ (Fig. 3a), which is for the aggregate reconfiguring from the $\mathrm{Lm}$ to Iso phase. To confirm that the shift originates from spatial redistribution of metal particles, absorption spectra of the NP assemblies were simulated using a multi-sphere scattering algorithm. The simulation was done with a program 

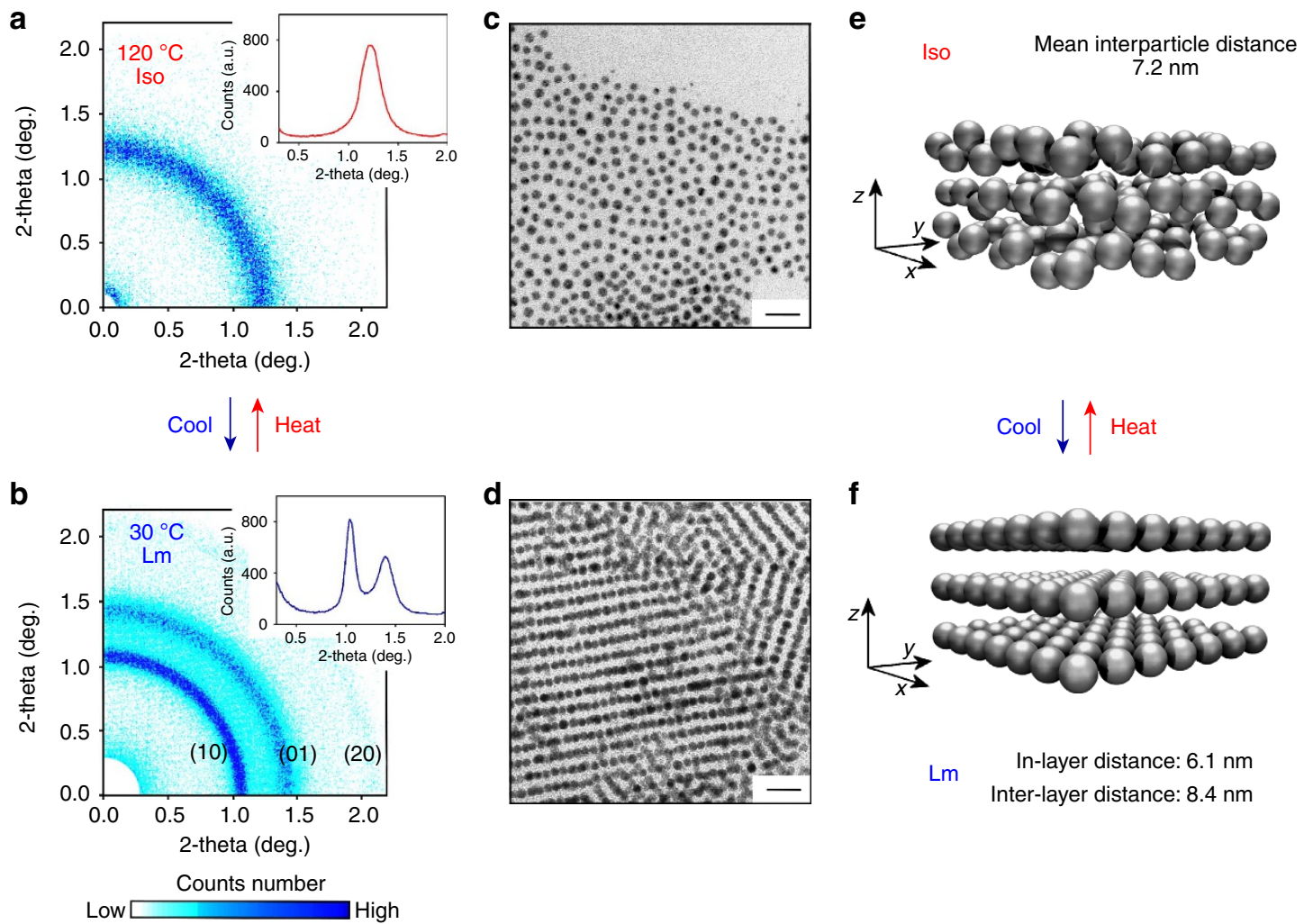

Lm In-layer distance: $6.1 \mathrm{~nm}$

Figure 2 | Structural analysis of Ag@L aggregate switching. (a) Representative SAXRD image of Ag@L aggregate at elevated (120 ${ }^{\circ} \mathrm{C}$ ) and (b) low $\left(30^{\circ} \mathrm{C}\right.$ ) temperatures, corresponding to isotropic (Iso) and lamellar $(\mathrm{Lm})$ arrangements, respectively; colour intensity map of count number is given below; insets show one-dimensional scans of the diffractograms. (c) Representative TEM image of thermally annealed Ag@L nanoparticles revealing patterns corresponding to the Iso and (d) Lm structures, scale bar, $20 \mathrm{~nm}$. (e) Scheme of nanoparticle arrangements in Iso and (f) Lm structures as deduced from SAXRD and TEM; organic ligands are omitted for clarity.

that solves self-consistently Maxwell's equations for an arbitrary arrangement of a finite number of spheres for a given illumination. This is achieved by expanding the incident, the scattered and the internal fields of each sphere into eigenmodes. The amplitudes of all eigenmodes can be calculated by enforcing the usual boundary conditions at the surface of the spheres. All further properties can be derived from these coefficients. To consider the actual structure in the simulations, the Lm phase was built from three layers composed of $17 \times 17$ metal particles with spacing as derived from SAXRD measurements, while the Iso structure was approximated by randomly shifting NP positions of the $\mathrm{Lm}$ structure to reach a mean nearest-neighbour distance of $7.2 \mathrm{~nm}$ according to the measurements (Fig. 3d). In preliminary simulations it was carefully verified that this number of silver spheres is fully sufficient to reflect the properties of an infinite sample, that is, all quantities of interest converged. The organic corona of NPs was treated as a homogenous medium with a refractive index of 1.6 , an intermediate value for LC molecules. A blue shift of $\lambda_{\max }$ was retrieved form the simulations of the optical spectra from 463 to $438 \mathrm{~nm}$ (Fig. 3b). This is slightly larger than the measured values, but nevertheless the wavelengths of plasmonic bands' maxima are in very good agreement with the experiment considering the complexity of the material (Fig. 3c). It is worth noting that for a structure similar to the Iso phase but with interparticle spacing corresponding to the nearest-neighbour distance in the $\mathrm{Lm}$ aggregate $(6.1 \mathrm{~nm})$, the retrieved $\lambda_{\max }$ was almost identical to the one for the $\mathrm{Lm}$ structure. This shall evidence that the nearest-neighbour distance variation between the $\mathrm{Lm}$ and Iso structures is the crucial parameter influencing plasmonic band position. This conclusion is further supported with a control experiment-no shift of plasmonic band position was observed for a heated sample of dodecanethiol-coated silver NPs (see Supplementary Fig. 6), for which no structural reconfiguration is observed within the given temperature limit.

To get a more precise picture of the influence of the spatial distribution of the NPs metallic cores on $\lambda_{\max }$, we have performed structural and optical measurements of Ag@L NPs heated in a stepwise manner (data collected every $10^{\circ} \mathrm{C}$, on heating, Fig. 3e,g). Up to $70^{\circ} \mathrm{C}$, the $\mathrm{Sm}$ phase is solely observed as evidenced by SAXRD but heating causes an expansion of the NP gap within the layers, while the interlayer distance reduces. This behaviour translates to a slow shift of $\lambda_{\max }$ towards smaller values. Then, in between 70 and $95^{\circ} \mathrm{C}$ a phase transition is observed from $\mathrm{Lm}$ to Iso phase-both structures coexist within this temperature range. The phase transition is accompanied with a faster kinetics of $\lambda_{\max }$ blue shift. Finally, above $95^{\circ} \mathrm{C}$ the Iso phase is solely found and the plasmonic band maximum reaches the lowest wavelength.

Evaluation of the switching mechanism. To understand the behaviour of promesogenic ligands during the heating process, we have performed DSC measurements of hybrid NPs with a heating rate of 5 deg. $\min ^{-1}$ (Fig. 3f). Two evident thermal events were identified taking place at ca. 60 and $90^{\circ} \mathrm{C}$. When compared with SAXRD results, it can be noticed that the first event can be associated with sharpening of the Bragg signal showing in-layer distance between NPs. Likewise, the second corresponds to the transition from $\mathrm{Lm}$ to Iso structure. It is therefore the melting of 

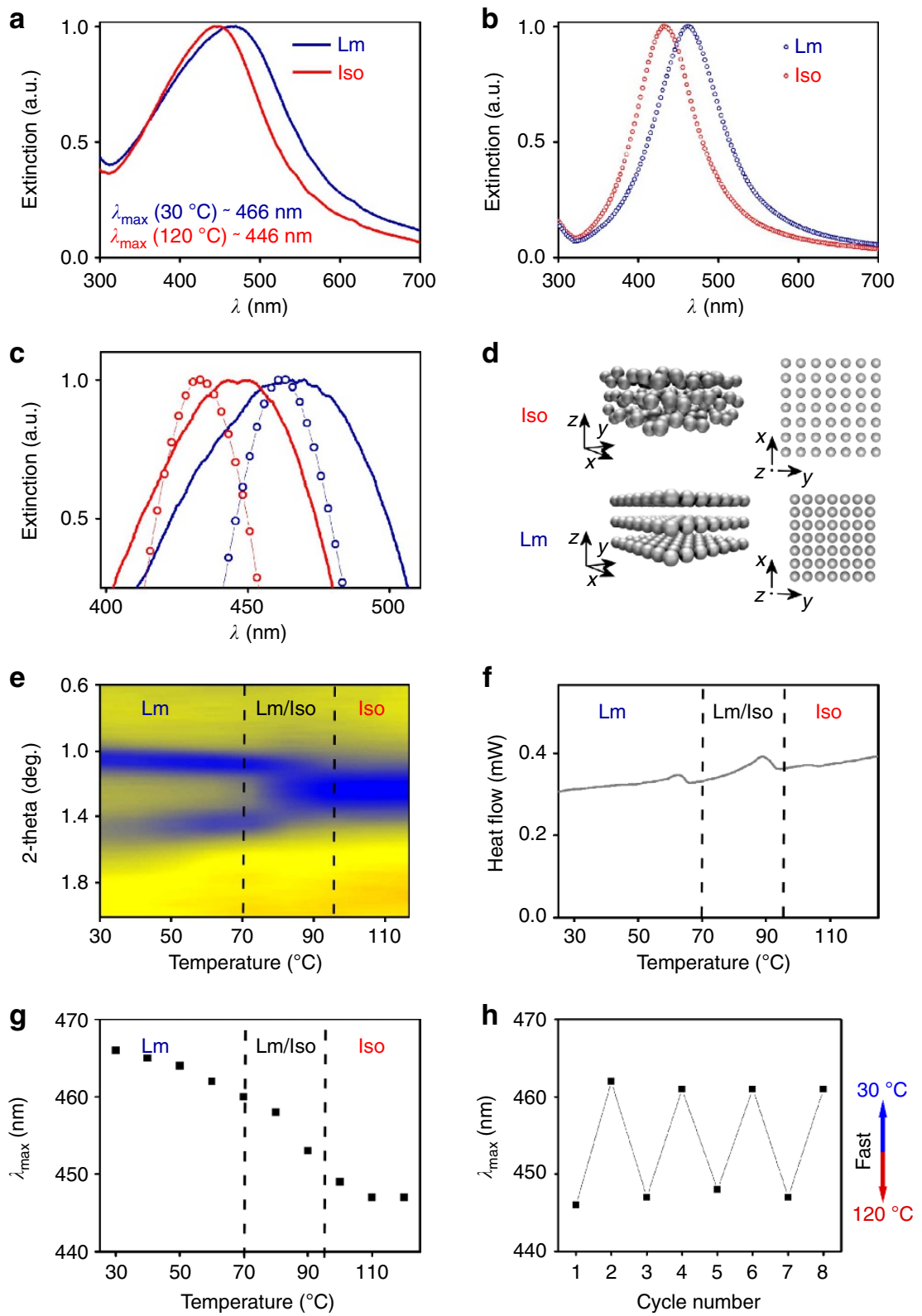

Figure 3 | Optical characterization of Ag@L aggregate. (a) Extinction spectra of the aggregate taken at 30 and $120^{\circ} \mathrm{C}$ revealing a shift of plasmon band maxima $\left(\lambda_{\max }\right)$. (b) Simulated extinction spectra of $\mathrm{Lm}$ and Iso structures. (c) Overlay of simulated and experimental extinction spectra. (d) Sketch of part of Iso and Lm aggregates (in fact layers comprised $17 \times 17$ particles) used in simulations. (e) Temperature-dependent SAXRD reflections evidencing regions of $\mathrm{Lm}, \mathrm{Lm} / \mathrm{Iso}$ and Iso structures' existence (divided by the dashed lines), collected on heating with a 10-deg. step. (f) DSC trace, collected on heating, of hybrid nanoparticles. Phase transition region in which $\mathrm{Lm} /$ Iso structures coexist is between the dashed lines. (g) Temperature-dependent $\lambda_{\text {max }}$ position shift, corresponding to the structural reconfiguration shown in $\mathbf{e}$. Phase transition region, as deduced based on $\mathbf{e}$, is between the dashed lines. (h) Reversible shifting of $\lambda_{\max }$ position in consecutive fast heating/cooling cycles.

$\mathrm{L}$ molecules at ca. $90^{\circ} \mathrm{C}$ that causes a rearrangement of silver NPs' metallic cores.

Kinetics and reversibility of switching. Next, we investigated the reversibility of Ag@L aggregate reconfiguration and examined the impact of the kinetics of temperature variation on the process. Both these parameters are important from the point of view of DSA and future applications. Noticeably, both structural and optical properties are fully reversible as attested by SAXRD and ultraviolet-visible measurements, but for this purpose slow cooling is needed. A well-developed $\mathrm{Lm}$ structure can be achieved by changing the temperature from 120 to $30^{\circ} \mathrm{C}$ in a stepwise manner with a $10^{\circ} \mathrm{C}$ step, which takes ca. $60 \mathrm{~min}$. Iso phase can be accessed more easily, still incubation in $120^{\circ} \mathrm{C}$ for few minutes is necessary to achieve the maximum plasmonic band shift. It would be advantageous to achieve fast switching behaviour; therefore, we tested the optical response of the sample to heating/ cooling cycles lasting only tens of seconds. SAXRD measurements of Ag@L aggregates cooled fast revealed coexistence of Lm and Iso phases (Supplementary Fig. 7). This translates to a lowered magnitude of plasmonic band shift in comparison with slow kinetics of cooling; a mean $\lambda_{\max }$ variation of ca. $14 \mathrm{~nm}$ was observed when the sample was quickly cycled between 120 and $30^{\circ} \mathrm{C}$ (Fig. 3h). Importantly, on the short term the tested hybrid NPs do not undergo decomposition at elevated temperatures as 
evidenced by $1 \mathrm{H}$ nuclear magnetic resonance (NMR) investigation (Supplementary Fig. 8); however, on the long term the system would probably slowly decompose due to oxidation of surface silver atoms ${ }^{54}$ causing release of stabilizing surface ligands.

Modelling metamaterial properties. The properties of the Ag@L NP aggregate were then considered in respect of achieving an ENZ regime. The effective dielectric function $\left(\varepsilon_{\text {eff }}\right)$ was calculated for Iso and Lm phases (Fig. 4a,b). The Clausius-Mossotti relation was used considering a particle in a lattice with the appropriate filling fraction corresponding to the respective interparticle distances. For the lamellar aggregate we used a second method, retrieving the effective properties from the complex reflection and transmission coefficients calculated assuming an infinitely extended rectangular lattice. We found very good agreement with the result from the Clausius-Mossotti relation. In both cases, the real part of $\varepsilon_{\text {eff }}$ exhibits both positive and negative regions due to
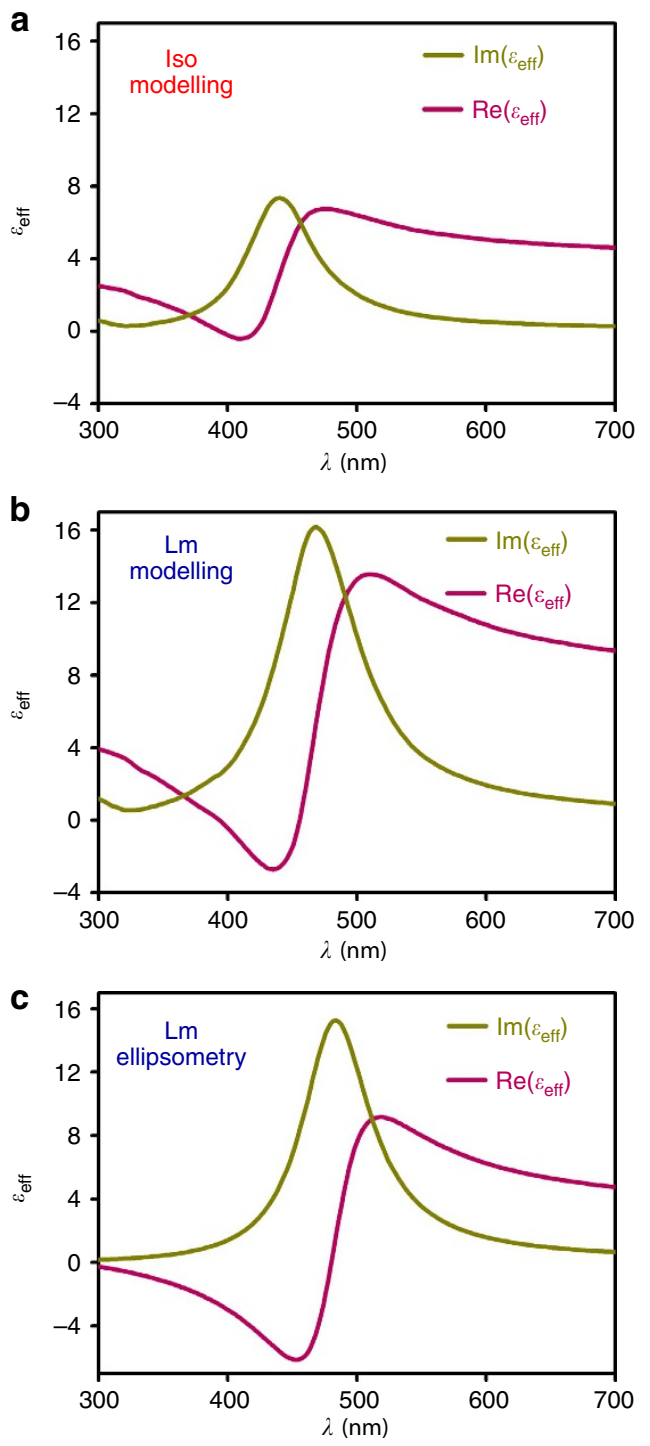

Figure 4 | Epsilon-near-zero properties of Ag@L material.

(a) Theoretically predicted imaginary $\left(\operatorname{Im}\left(\varepsilon_{\text {eff }}\right)\right)$ and real $\left(\operatorname{Re}\left(\varepsilon_{\text {eff }}\right)\right)$ parts of the dielectric function of Ag NP aggregates in the Iso and (b) Lm structure. (c) Dielectric functions calculated based on spectroscopic ellipsometry measurements. a strong Lorentzian resonance centred at the collective surface plasmon polariton resonance. Such properties are characteristic to transparent and metallic materials, respectively. Importantly, the real part of the permittivity reaches zero, allowing us to classify this material as ENZ, for Lm and Iso structures at slightly different wavelengths. For an actual application, the short-wavelength regime is very likely attractive since it is off-resonant and propagating light would therefore experience lower absorption. It should be also noted that, for modelling purposes small monodomains of $\mathrm{Lm}$ and Iso aggregates are considered. This should be fully sufficient to describe the global arrangement properties, since the monodomains are optically comparable and no nonlocal effective properties are observed in the process of homogenization. This strongly supports the idea that the material is an actively tunable metamaterial.

To determine the bandwidths of the ENZ properties of the Ag@L material in the Lm and Iso phases, various criteria can be considered as introduced previously by other authors $8,54,64,65$. Rough estimation, for example, based on identifying the spectral range where $\operatorname{Re}\left(\varepsilon_{\text {eff }}\right)$ values are in between -1.0 and 1.0 yields ENZ properties for wavelengths' ranges 365-410 nm for $\mathrm{Lm}$, and $375-430 \mathrm{~nm}$ for Iso structures (Supplementary Fig. 9) ${ }^{8,54}$. Naturally, narrower ENZ regions are retrieved if $\operatorname{Re}\left(\varepsilon_{\mathrm{eff}}\right)$ values are restricted to the -0.2 to 0.2 range as used by Pinchuk et al. ${ }^{64}$, yet tunability is still observed (Supplementary Fig. 9). Obviously, the real part of the permittivity is exactly equal to zero only at a single wavelength. We wish to stress that the effect we exploit is resonant, which means that dispersion is always accompanied by absorption. Although we work at off-resonant frequencies, absorption remains notable. Following suggestions from literature $^{65}$, we quantify this influence using a figure of merit (FOM) defined according to equation (1):

$$
\operatorname{Re}\left(n_{\text {eff }}\right) / 2 \operatorname{Im}\left(n_{\text {eff }}\right)
$$

Equation (1) expresses the propagation length of light in the ENZ material in units of the wavelength. Using the permittivity values retrieved from the simulations a FOM of $\sim 0.5$ is found at the wavelength where the real part of epsilon is zero. In the ENZ window of the $\mathrm{Lm}$ structure $(365-410 \mathrm{~nm})$, the propagation length of light takes values between 0.4 and 1.2 wavelengths. For the Iso structure the extreme values of FOM in the ENZ region are 0.4 and 1.0 .

To further confirm ENZ properties of the NP assembly, we have performed variable wavelength ellipsometric spectroscopy of a thin film of Ag@L material on a silicone substrate (details of the measurements are given in the Methods). Prior to the measurements the material was thermally annealed to assure Lm arrangement of NPs and the thickness of the film was measured using atomic force microscopy (AFM) (Supplementary Fig. 10). For the purpose of fitting the acquired ellipsometric data (amplitude and phase of reflected light versus light wavelength, Supplementary Fig. 11), we rely on a complex function for the effective permittivity based on two assumptions-that the material is made from small, spherical particles made of silver (properties of which can be reflected by a permittivity function for Ag that is built in the software) embedded into an organic matter with aromatic moieties (properties thereof are reflected by a harmonic oscillator with free parameters). It should be noted that similar results were obtained when using an effective medium approach with Bruggeman analysis, approximating the medium as a mixture of aromatic molecules (reflected by an oscillator) and silver (reflected by the built-in permittivity for $\mathrm{Ag})^{2,64}$. Similar approaches have been applied successfully in the past to describe effective properties of composites with a comparable topography ${ }^{2}$. The outlined procedure enabled us to retrieve macroscopic, dielectric functions of the Ag@L sample 
having Lm order (Fig. 4c, for real and imaginary parts of refractive index see Supplementary Fig. 12), which are in very good agreement with the theoretically predicted properties.

\section{Discussion}

In the outlined paper we have achieved DSA of silver NPs for which metamaterial properties were simulated and verified by ellipsometry measurements. LC ligands grafted onto the metallic surface were used to drive the reconfiguration of the aggregate from a lamellar to an isotropic structure. Variation of spatial distribution of NPs allows engineering the nearest-neighbour interparticle gap in the 2.5-1.4-nm range and results from the changes of the geometry of the organic coating layer. This translates to switching of the aggregate plasmonic band position by $20 \mathrm{~nm}$ and allows active tunability of the materials dielectric functions. Notably, the relatively thick organic coating results in a metal volume fraction suited for the observation of ENZ properties. The ENZ properties are observed for wavelengths in between 365 and $430 \mathrm{~nm}$, which is especially interesting due to the need for metamaterials working in the visible range for future optical electronics technologies. Importantly, the spectral regions for Iso and Lm structures are slightly different, confirming tunability of the metamaterial properties. Another key feature of the presented system is that the observed switching behaviour of the material is fully reversible both in the view of structure and properties if slow cooling is used. Fast changes of temperature also result in a change of the optical response but with a slightly decreased magnitude of the plasmonic band shift.

The approach to reconfigurable metamaterials presented here provides opportunities, but it also puts some challenges. The range of the tunability is limited due to the use of large surface ligands, therefore profound changes of the physicochemical characteristic (for example, dielectric-to-metal transition) of the material can hardly be achieved since interparticle gaps smaller than $0.5 \mathrm{~nm}$ are necessary to assure strong interparticle coupling. This problem can be addressed either by minimizing the lowest interparticle gap or assembling larger silver particles, keeping the thickness of the organic coating constant. Achieving these goals would be non-trivial, however, it should be noted that both unusually low interparticle spacing ${ }^{37}(\mathrm{ca} .1 \mathrm{~nm})$ and assembly of larger $\mathrm{NPs}^{40}$ (ca. $\left.10 \mathrm{~nm}\right)$ using the outlined strategy have been reported. Proper design of surface ligands should also allow to lower the phase transition temperature, assuring long-term stability of the material. The final challenge is kinetics of the reconfiguration. The current state-of-the-art are switching times on the scale of microseconds achieved by Zheludev et al. ${ }^{20}$, which are beyond the scope of our approach. The presented material allows for reconfiguration on the scale of tens of seconds, comparable to other systems based on DSA of nanocrystals; however, the use of liquid crystals as the driving force for rearrangement allows one to predict that tunability on shorter timescales will be achievable.

In summary, the strategy presented here achieves tunability of a NP aggregate with ENZ properties evidencing that nextgeneration materials with adaptive behaviour can be obtained via the LC approach.

\footnotetext{
Methods

Materials and equipment. Solvents and substrates were obtained from SigmaAldrich. Before use solvents were dried over activated molecular sieves for $24 \mathrm{~h}$. Substrates were used without further purification. All reactions were carried out under nitrogen $\left(\mathrm{N}_{2}\right)$ atmosphere in dried glassware and assuring efficient magnetic stirring. Purification of reaction products was carried out by column chromatography using RushanTaiyang silica gel 60 (230-400 mesh) at atmospheric pressure or by crystallization if possible. Analytical thin-layer chromatography was performed using a Silica Gel $60 \AA$ F254 (Merck) pre-coated glass plater $(0.25 \mathrm{~mm}$ thickness) and visualized using iodine vapour and/or an ultraviolet lamp $(254 \mathrm{~nm})$.
}

Yields refer to chromatographically and spectroscopically $\left({ }^{1} \mathrm{H}\right.$ NMR) homogeneous materials.

The ${ }^{1} \mathrm{H}$ NMR and ${ }^{13} \mathrm{C}$ NMR spectra were recorded at either 200 or $500 \mathrm{MHz}$ NMR Varian Unity Plus. Proton chemical shifts are reported in p.p.m. $(\delta)$ relative to the internal standard-tetramethylsilane $(\delta=0.00$ p.p.m.). Carbon chemical shifts are reported in p.p.m. $(\delta)$ relative to the residual solvent signal $\left(\mathrm{CDCl}_{3}\right.$, $\delta=77.0$ p.p.m.). Data are presented as follows: chemical shift, integration, multiplicity $(\mathrm{s}=$ singlet, $\mathrm{d}=$ doublet, $\mathrm{t}=$ triplet, $\mathrm{q}=$ quartet, $\mathrm{br}=$ broad and $\mathrm{m}=$ multiplet), and coupling constant $(\mathrm{Hz})$. In all recorded spectra there are also sharp signals coming from a small amount of known impurities present in used solvent: $\mathrm{H}_{2} \mathrm{O}$ (1.7 p.p.m.) and $\mathrm{CHCl}_{3}$ (7.27 p.p.m.).

SAXRD patterns for the powder as well as partially aligned samples were obtained with the BrukerNanostar system. The $\mathrm{CuK} \alpha$ radiation was used; patterns were registered with an area detector VANTEC2000. The temperature of the sample was controlled with a precision of $0.1 \mathrm{~K}$. Kapton tape was used as a substrate for NP measurements.

XPS experiments were performed in a PHl 5,000 VersaProbe-Scanning ESCA microprobe (ULVAC-PHI, Japan/USA) instrument at a base pressure below $5 \times 10^{-9} \mathrm{mbar}$. Monochromatic $\mathrm{Al} \mathrm{K \alpha}$ radiation was used and the X-ray beam, focused to a diameter of $100 \mu \mathrm{m}$, was scanned on a $250 \times 250-\mu \mathrm{m}$ surface, at an operating power of $25 \mathrm{~W}$. Photoelectron survey spectra were acquired using a hemispherical analyzer at pass energy $117.4 \mathrm{eV}$ with a $0.4-\mathrm{eV}$ energy step; core-level spectra were acquired at pass energy $23.5 \mathrm{eV}$ with a $0.1-\mathrm{eV}$ energy step. All spectra were acquired with $90^{\circ}$ between X-ray source and analyzer and with the use of lowenergy electrons and low-energy argon ions for charge neutralization. After subtraction of the Shirley-type background, the core-level spectra were decomposed into their components with mixed Gaussian-Lorentzian (30:70) shape lines using the CasaXPS software. Quantification calculations were conducted using sensitivity factors supplied by PHI. Spectra were calibrated against $284.6 \mathrm{eV}$ for C $1 s$ region.

Transmission electron micrography was performed using a Zeiss Libra 120 microscope, with LaB6 cathode, equipped with OMEGA internal columnar filters and a charge-coupled device camera.

TGA were performed with a TA Q50 V20.13 (TA Instruments) analyzer. The measurements were carried out in the $20-600-{ }^{\circ} \mathrm{C}$ range with $10 \mathrm{~K} \mathrm{~min}^{-1}$ heating rate in air.

The ultraviolet-visible spectrum was measured using a Cary 5,000 spectrometer (Agilent). The solutions of functionalized particles in dichloromethane were held in standard poly(methyl methacrylate) (PMMA) cuvettes (VWR) with a 10-mm optical path, while the aggregates were recorded on a quartz substrate in a transmission mode.

TEM images were taken using the Zeiss Libra 120 microscope.

Ellipsometric measurements were made using a variable-angle spectroscopic ellipsometer SENTECH SE850 (SENTECH Instruments GmbH). The acquired data were analysed using SpectraRay II software package.

AFM measurements were performed in tapping mode with a Dimension Icon (Bruker) microscope using SCANASYST-AIR tip (silicon tip on nitride lever).

NP synthesis. Silver clusters with dodecanethiol coating were prepared as previously described ${ }^{25}$ using a modified literature method ${ }^{37}$. Fifty $\mathrm{ml}$ of dodecylamine $(1.5 \mathrm{~g})$ solution in cyclohexane $(50 \mathrm{ml})$ was stirred for $10 \mathrm{~min}$ with $12 \mathrm{ml}$ aqueous formaldehyde (37\%). The organic phase was separated out and washed twice with water $(2 \times 50 \mathrm{ml})$. Then, aqueous solution of $\mathrm{AgNO}_{3}\left(0.4 \mathrm{~g} \mathrm{AgNO}_{3}\right.$ in $\left.20 \mathrm{ml} \mathrm{H}_{2} \mathrm{O}\right)$ was added, left to stir for $40 \mathrm{~min}$ after which dodecanethiol $(0.3$ molar eq. of dodecylamine) was added and the reaction was stirred overnight. Organic phase was separated and NPs were precipitated by addition of $100 \mathrm{ml}$ of ethanol. The precipitate was centrifuged, collected, dissolved in a small amount of cyclohexane $(10 \mathrm{ml})$ and again the precipitation procedure was repeated. The obtained NPs (Ag@ $\left.\mathrm{C}_{12} \mathrm{H}_{25} \mathrm{SH}\right)$ were used as the starting material for the preparation of hybrid NPs, denoted as Ag@L. To $15 \mathrm{mg}$ of NPs dissolved in $10 \mathrm{ml}$ hexane/

dichloromethane (DCM) mixture $(v / v=1 / 1) 20 \mathrm{mg}$ of the L ligand was added. The reaction proceeded at room temperature for $24 \mathrm{~h}$. Then, NPs were precipitated with $20 \mathrm{ml}$ of methanol and centrifuged (13,000 r.p.m., $5 \mathrm{~min}$ ). Supernatant containing unbound thiol ligands was discarded. The precipitate was dissolved in $1 \mathrm{ml}$ of warm hexane and centrifuged after cooling down; then, the process was repeated. This washing procedure was repeated until no traces of free mesogenic ligand remained, as determined by thin-layer chromatography.

Thermogravimetric analysis of NPs. To evaluate the exact composition of organic coronas of the studied NPs, TGA was used. The weight loss for dodecanethiol-coated silver NPs (Ag@ $\mathrm{C}_{12} \mathrm{H}_{25} \mathrm{SH}$, below $260^{\circ} \mathrm{C}$ ) was $23.40 \%$. This value was attributed to the removal of the organic shell and recalculated to the number of surface alkyl thiols as outlined below. TGA traces for hybrid NPs (Ag@L) revealed substantially larger weight losses in two distinct steps. The first one, below $260^{\circ} \mathrm{C}$ can be attributed to the removal of alkyl thiol molecules and accounts for a mass drop of $12 \%$. A wider peak at higher temperature values $\left(>260^{\circ} \mathrm{C}\right)$ is due to the removal of $\mathrm{L}$ molecules (weight loss $35.37 \%)$. The numbers of alkyl $\left(N_{\text {alkyl }}\right)$ and mesogenic ligands $\left(N_{\mathrm{L}}\right)$ were calculated as given below.

To convert the mass loss measured by the TGA measurements to surface ligand stoichiometry, we first calculated the mass of single metal spheres for silver $\left(M_{\mathrm{Ag}}\right)$ 
NPs, using the diameter derived from SAXS and TEM and the bulk density of metals $\rho_{\mathrm{Ag}}=10.49 \mathrm{~g} \mathrm{~cm}^{-3}$. The calculated mass was: $M_{\mathrm{Ag}}=5.7 \times 10^{-19} \mathrm{~g}$. The mass of organic matter $\left(M_{\text {org }}\right)$ removed from a single NP was calculated using $\%$ of mass left after the analysis ( $\left.\% M_{\text {left }}\right)$ and $\%$ of mass loss ( $\left.\% M_{\text {loss }}\right)$ using equation (2):

$$
M_{\text {org }}=M_{\mathrm{Ag}(\mathrm{orAu})} /\left(\% M_{\text {left }} \times \% M_{\text {loss }}\right) . \% M_{\text {left }} \text { and } \% M_{\text {loss }}
$$

can be read out of Supplementary Fig. 4a. To retrieve the number of ligands per NP, $M_{\text {org }}$ has to be divided by the mass (in (g)) of the ligand responsible for the given mass drop: $m_{\mathrm{C} 12 \mathrm{H} 25 \mathrm{SH}}=3.36 \times 10^{-22} \mathrm{~g}, m_{\mathrm{L}}=1.37 \times 10^{-21} \mathrm{~g}$. For NPs after the exchange reaction mass losses below and above $260^{\circ} \mathrm{C}$ were treated separately.

The number of alkyl ligands in $\mathrm{Ag} @ \mathrm{C}_{12} \mathrm{H}_{25} \mathrm{SH}: 520$.

The number of alkyl ligands in Ag@L: 240.

The number of L ligands in Ag@L: 260.

Numerical calculations. The numerical calculations were performed with an inhouse code introduced in ref. 66. For the metal parameters, the data of Johnson and Christy with a size correction for the imaginary part were used; this is necessary as soon as the particles are much smaller than the wavelength, that is, especially when they are small relative to the mean free path of the electrons in a metal. To simulate a reasonable chunk of matter a structure of three shifted layers of 17 times 17 silver spheres with radius $2.35 \mathrm{~nm}$, as it was found in the TEM measurements, was considered for the extinction spectra of the Lm phase. This was sufficient to let all interesting properties converge. The layers were separated by $8.6 \mathrm{~nm}$ and the spheres were placed on a square grid with a period of $6.1 \mathrm{~nm}$ according to the SAXRD and TEM measurements. To simulate the Iso phase, all spheres of the previous structure were shifted by a random amount in a random direction to negate the long-range order of the structure and to achieve a nearest-neighbour distance of $7.2 \mathrm{~nm}$.

To get the effective material properties of the Iso phase the Clausius-Mossotti relation was used. Therefore, the polarizability of a single silver sphere was computed and the filling factor as extracted from the SAXRD measurements was used to calculate the effective permittivity. We used a second code capable of calculating the reflection and transmission coefficients of three layers of an infinitely extended lattice, which is a good representation of the lamellar structure. Again the interparticle and interlayer distances from the SAXRD and TEM measurements of the Lm phase were used. From these reflection and transmission coefficients the effective permittivity was calculated. To cross-check the methods, we also used the Clausius-Mossotti relation using a filling factor corresponding to the Lm phase and a very good agreement was found as is shown in Supplementary Fig. 13.

Bandwidths of the ENZ properties of the Ag@L material were retrieved from identifying the spectral range where $\operatorname{Re}\left(\varepsilon_{\text {eff }}\right)$ values are in between -1.0 and 1.0 (Supplementary Fig. 9). On the basis of the retrieved values of effective permittivity, real and imaginary parts of refractive index were calculated, which allowed us to calculate FOM defined as $\operatorname{Re}\left(n_{\text {eff }}\right) / 2 \operatorname{Im}\left(n_{\text {eff }}\right)$.

Ellipsometric measurements. For the ellipsometric measurements, a thin film of Ag@L NPs in toluene/chloroform $(v / v=9: 1)$ mixture was spin casted onto two types of substrates: boron-doped silicone wafer substrate cut along [911] plane (CEMAT SILICONE S.A.) and alumina. The substrates with the NP film were then heated to $120^{\circ} \mathrm{C}$ and cooled slowly to room temperature in the manner corresponding to samples prepared for SAXRD measurements, to assure the $\mathrm{Lm}$ structure of the aggregate. Then, the thicknesses of the samples were measured using AFM, showing relatively low height variance. Amplitude (Psi) and phase (Delta) of the complex reflectance ratio were measured for wavelengths from 400 to $800 \mathrm{~nm}$ with a $2-\mathrm{nm}$ step, at $70^{\circ}, 65^{\circ}$ and $60^{\circ}$ angle of incidence. Data were fitted using SpectraRay II software package (SENTECH Instruments GmbH) by minimizing the square root of variance. To fit the substrates software built-in functions for $\mathrm{Si}$ and $\mathrm{Al}$ covered with $\mathrm{Al}_{2} \mathrm{O}_{3}$ were used, achieving parameters in agreement with the literature values. For the purpose of fitting the acquired ellipsometric data (amplitude and phase of reflected light versus light wavelength, Supplementary Fig. 11), we rely on a complex function for the effective permittivity based on two assumptions - that the material is made from small, spherical particles made of silver (properties of which can be reflected by a permittivity function for Ag that is built in the software) embedded into an organic matter with aromatic moieties (properties thereof are reflected by a harmonic oscillator with free parameters). It should be noted that similar results were obtained when using an effective medium approach with Bruggeman analysis, approximating the medium as a mixture of aromatic molecules (reflected by an oscillator) and silver (reflected by the built-in permittivity for $\mathrm{Ag}$ ). Imaginary part of $\varepsilon_{\text {inf }}$ was set to 0 and not fitted. We have taken care to find the global minimum for fitting the data by large, random changes of the function parameter values. A sample result characteristic to a whole set of samples is given in Supplementary Fig. 11 (Si substrate). AFM measurement of film thickness for the same sample (ca. $100 \mathrm{~nm}$ ) is given in Supplementary Fig. 10. The data taken for different samples exhibit similar characteristics with an anomaly occurring below $500 \mathrm{~nm}$, but slightly differing depending on the film thickness, roughness and uniformity of the sample. The mean square error of the fitting shown in Supplementary Fig. 11 is 1.1.

\section{References}

1. Fruhnert, M., Mühlig, S., Lederer, F. \& Rockstuhl, C. Towards negative index self-assembled metamaterials. Phys. Rev. B 89, 075408 (2014).

2. Fafarman, A. T. et al. Chemically tailored dielectric-to-metal transition for the design of metamaterials from nanoimprinted colloidal nanocrystals. Nano Lett. 13, 350-357 (2013).

3. Liu, Q. et al. Self-alignment of plasmonic gold nanorods in reconfigurable anisotropic fluids for tunable bulk metamaterial applications. Nano Lett. 10, 1347-1353 (2010).

4. Mühlig, S. et al. Self-assembled plasmonic metamaterials. Nanophotonics 2, 1-30 (2013)

5. Malassis, L. et al. Topological darkness in self-assembled plasmonic metamaterials. Adv. Mater. 26, 324-330 (2014).

6. Alisafaee, H. \& Fiddy, M. A. Polarization insensitivity in epsilon-near-zero metamaterial from plasmonic aluminum-doped zinc oxide nanoparticles. J. Nanophoton. 8, 083898 (2014).

7. Fontana, J., Naciri, J., Rendell, R. \& Ratna, B. R. Macroscopic self-assembly and optical characterization of nanoparticle-ligand metamaterials. Adv. Opt. Mater. 1, 100-106 (2013).

8. Young, K. L. et al. Using DNA to design plasmonic metamaterials with tunable optical properties. Adv. Mater. 26, 653-659 (2014).

9. Shafiei, F. et al. A subwavelength plasmonic metamolecule exhibiting magneticbased optical Fano resonance. Nat. Nanotechnol. 8, 95-99 (2013).

10. Moreau, A. et al. Controlled-reflectance surfaces with film-coupled colloidal nanoantennas. Nature 492, 86-89 (2012).

11. Tamma, V. A., Cui, Y. \& Park, W. Scattering reduction at near-infrared frequencies using plasmonic nanostructures. Opt. Express 21, 1041-1056 (2013).

12. Mühlig, S. et al. A self-assembled three-dimensional cloak in the visible. Sci. Rep. 3, 2328 (2013).

13. Paik, T. et al. Solution-processed phase-change $\mathrm{VO}(2)$ metamaterials from colloidal vanadium oxide (VO(x)) nanocrystals. ACS Nano 8, 797-806 (2014)

14. Kruger, B. a., Joushaghani, A. \& Poon, J. K. S. Design of electrically driven hybrid vanadium dioxide (VO2) plasmonic switches. Opt. Express 20, 23598-23609 (2012).

15. Dicken, M. J. et al. Frequency tunable near-infrared metamaterials based on VO2 phase transition. Opt. Express 17, 18330-18339 (2009).

16. Driscoll, T. et al. Dynamic tuning of an infrared hybrid-metamaterial resonance using vanadium dioxide. Appl. Phys. Lett. 93, 024101 (2008).

17. Shin, D. et al. Broadband electromagnetic cloaking with smart metamaterials. Nat. Commun. 3, 1213 (2012).

18. Zhao, C., Liu, Y., Zhao, Y., Fang, N. \& Huang, T. J. A reconfigurable plasmofluidic lens. Nat. Commun. 4, 2305 (2013).

19. Jung, P. et al. Multistability and switching in a superconducting metamaterial. Nat. Commun. 5, 3730 (2014).

20. Ou, J.-Y., Plum, E., Zhang, J. \& Zheludev, N. I. An electromechanically reconfigurable plasmonic metamaterial operating in the near-infrared. Nat. Nanotechnol. 8, 252-255 (2013).

21. Buchnev, O., Ou, J. Y., Kaczmarek, M., Zheludev, N. I. \& Fedotov, V. A. Electro-optical control in a plasmonic metamaterial hybridised with a liquidcrystal cell. Opt. Express 21, 1633-1638 (2013).

22. Gil, I., Bonache, J., Garcia-Garcia, J. \& Martin, F. Tunable metamaterial transmission lines based on varactor-loaded split-ring resonators. IEEE Trans. Microw. Theory Tech. 54, 2665-2674 (2006).

23. Zhao, Q. et al. Electrically tunable negative permeability metamaterials based on nematic liquid crystals. Appl. Phys. Lett. 90, 011112 (2007).

24. Kim, D. K., Lai, Y., Diroll, B. T., Murray, C. B. \& Kagan, C. R. Flexible and lowvoltage integrated circuits constructed from high-performance nanocrystal transistors. Nat. Commun. 3, 1216 (2012).

25. Nag, A. et al. Metal-free inorganic ligands for colloidal nanocrystals: $\mathrm{S}^{2-}$, $\mathrm{HS}^{-}, \mathrm{Se}^{2-}, \mathrm{HSe}^{-}, \mathrm{Te}^{2-}, \mathrm{HTe}^{-}, \mathrm{TeS}_{3}^{2-}, \mathrm{OH}^{-}$, and $\mathrm{NH}^{2-}$ as surface ligands. J. Am. Chem. Soc. 133, 10612-10620 (2011).

26. Dong, A., Jiao, Y. \& Milliron, D. Electronically coupled nanocrystal superlattice films by in situ ligand exchange at the liquid-air interface. ACS Nano 7, 10978-10984 (2013).

27. Koh, W., Saudari, S. R., Fafarman, A. T., Kagan, C. R. \& Murray, C. B. Thiocyanate-capped $\mathrm{PbS}$ nanocubes: ambipolar transport enables quantum dot based circuits on a flexible substrate. Nano Lett. 11, 4764-4767 (2011).

28. Talapin, D. V. \& Murray, C. B. PbSe nanocrystal solids for n- and p-channel thin film field-effect transistors. Science 310, 86-89 (2005).

29. Ross, M. B., Blaber, M. G. \& Schatz, G. C. Using nanoscale and mesoscale anisotropy to engineer the optical response of three-dimensional plasmonic metamaterials. Nat. Commun. 5, 4090 (2014).

30. Stratford, K., Henrich, O., Lintuvuori, J. S., Cates, M. E. \& Marenduzzo, D. Selfassembly of colloid-cholesteric composites provides a possible route to switchable optical materials. Nat. Commun. 5, 3954 (2014).

31. Young, K. L. et al. Assembly of reconfigurable one-dimensional colloidal superlattices due to a synergy of fundamental nanoscale forces. Proc. Natl Acad. Sci. USA 109, 2240-2245 (2012). 
32. Sashuk, V. et al. Nanoparticles in a capillary trap: dynamic self-assembly at fluid interfaces. ACS Nano 7, 8833-8839 (2013).

33. Lagzi, I., Kowalczyk, B., Wang, D. \& Grzybowski, B. A. Nanoparticle oscillations and fronts. Angew. Chem. Int. Ed. Engl. 49, 8616-8619 (2010).

34. Chovnik, O., Balgley, R., Goldman, J. R. \& Klajn, R. Dynamically selfassembling carriers enable guiding of diamagnetic particles by weak magnets. J. Am. Chem. Soc. 134, 19564-19567 (2012).

35. Tao, A., Sinsermsuksakul, P. \& Yang, P. Tunable plasmonic lattices of silver nanocrystals. Nat. Nanotechnol. 2, 435-440 (2007).

36. Collier, C. P. Reversible tuning of silver quantum dot monolayers through the metal-insulator transition. Science 277, 1978-1981 (1997).

37. Dintinger, J. et al. A self-organized anisotropic liquid-crystal plasmonic metamaterial. Adv. Mater. 25, 1999-2004 (2013).

38. Lewandowski, W. et al. Smectic mesophases of functionalized silver and gold nanoparticles with anisotropic plasmonic properties. Chem. Commun. 49, 7845-7847 (2013).

39. Kanie, K. et al. Simple cubic packing of gold nanoparticles through rational design of their dendrimeric corona. J. Am. Chem. Soc. 134, 808-811 (2012).

40. Yu, C. H. et al. Design, synthesis, and characterization of mesogenic aminecapped nematic gold nanoparticles with surface-enhanced plasmonic resonances. J. Am. Chem. Soc. 134, 5076-5079 (2012).

41. Draper, M. et al. Self-assembly and shape morphology of liquid crystalline gold metamaterials. Adv. Funct. Mater. 21, 1260-1278 (2011).

42. Mischler, S., Guerra, S. \& Deschenaux, R. Design of liquid-crystalline gold nanoparticles by click chemistry. Chem. Commun. 48, 2183-2185 (2012).

43. Lewandowski, W., Wójcik, M. \& Górecka, E. Metal nanoparticles with liquidcrystalline ligands: controlling nanoparticle superlattice structure and properties. Chemphyschem 15, 1283-1295 (2014).

44. Nealon, G. L. et al. Liquid-crystalline nanoparticles: hybrid design and mesophase structures. Beilstein J. Org. Chem. 8, 349-370 (2012).

45. Bisoyi, H. K. \& Kumar, S. Liquid-crystal nanoscience: an emerging avenue of soft self-assembly. Chem. Soc. Rev. 40, 306-319 (2011).

46. Umadevi, S., Feng, X. \& Hegmann, T. Large area self-assembly of nematic liquid-crystal-functionalized gold nanorods. Adv. Funct. Mater. 23, 1393-1403 (2013).

47. Wojcik, M. M. et al. Temperature-controlled liquid crystalline polymorphism of gold nanoparticles. Soft Matter 7, 10561-10564 (2011).

48. Zhao, Y. et al. Small-molecule-directed nanoparticle assembly towards stimuliresponsive nanocomposites. Nat. Mater. 8, 979-985 (2009).

49. Li, Q. et al. Responsive assemblies: gold nanoparticles with mixed ligands in microphase separated block copolymers. Adv. Mater. 20, 1462-1466 (2008).

50. Heo, K., Miesch, C., Emrick, T. \& Hayward, R. C. Thermally reversible aggregation of Gold nanoparticles in polymer nanocomposites through hydrogen bonding. Nano Lett. 13, 5297-5302 (2013).

51. Tokarev, I. \& Minko, S. Tunable plasmonic nanostructures from noble metal nanoparticles and stimuli-responsive polymers. Soft Matter 8, 5980-5987 (2012).

52. Schreiber, R. et al. Chiral plasmonic DNA nanostructures with switchable circular dichroism. Nat. Commun. 4, 2948 (2013).

53. Yu, X. et al. Distance control in-between plasmonic nanoparticles via biological and polymeric spacers. Nano Today 8, 480-493 (2013).

54. Maas, R., Parsons, J., Engheta, N. \& Polman, A. Experimental realization of an epsilon-near-zero metamaterial at visible wavelengths. Nat. Photon. 7, 907-912 (2013).

55. Engheta, N. Pursuing near-zero response. Science 340, 286-287 (2013).
56. Alù, A., Silveirinha, M., Salandrino, A. \& Engheta, N. Epsilon-near-zero metamaterials and electromagnetic sources: tailoring the radiation phase pattern. Phys. Rev. B 75, 155410 (2007).

57. Rycenga, M. et al. Controlling the synthesis and assembly of silver nanostructures for plasmonic applications. Chem. Rev. 111, 3669-3712 (2011).

58. Chen, Y. \& Wang, X. Novel phase-transfer preparation of monodisperse silver and gold nanoparticles at room temperature. Mater. Lett. 62, 2215-2218 (2008).

59. Lewandowski, W., Jatczak, K., Pociecha, D. \& Mieczkowski, J. Control of gold nanoparticle superlattice properties via mesogenic ligand architecture. Langmuir 29, 3404-3410 (2013).

60. Donnio, B., García-Vázquez, P., Gallani, J.-L., Guillon, D. \& Terazzi, E. Dendronized ferromagnetic gold nanoparticles self-organized in a thermotropic cubic phase. Adv. Mater. 19, 3534-3539 (2007).

61. Weissflog, W. et al. Ten isomeric five-ring bent-core mesogens: The influence of the direction of the carboxyl connecting groups on the mesophase behaviour J. Mater. Chem. 15, 4328-4337 (2005).

62. Wojcik, M. et al. Liquid-crystalline phases made of gold nanoparticles. Angew. Chem. Int. Ed. Engl. 48, 5167-5169 (2009)

63. Nguyen, T. D., Jankowski, E. \& Glotzer, S. C. Self-assembly and reconfigurability of shape-shifting particles. ACS Nano 5, 8892-8903 (2011).

64. Goncharenko, A. V., Venger, E. F., Chang, Y. C. \& Pinchuk, A. O. Arrays of core-shell nanospheres as $3 \mathrm{~d}$ isotropic broadband ENZ and highly absorbing metamaterials. Opt. Mater. Express 4, 2310-2322 (2014).

65. Traviss, D., Bruck, R., Mills, B., Abb, M. \& Muskens, O. L. Ultrafast plasmonics using transparent conductive oxide hybrids in the epsilon-near-zero regime. Appl. Phys. Lett. 102, 121112 (2013).

66. Mühlig, S. et al. Three-dimensional metamaterial nanotips. Phys. Rev. B 81, 075317 (2010).

\section{Acknowledgements}

W.L. would like to acknowledge support by the Polish National Science Center (Project 2013/09/D/ST5/03875). M.F. and C.R. would like to acknowledge support by the German Federal Ministry of Education and Research (PhoNa) and by the Thuringian State Government (MeMa). W.L. and E.G. would like to acknowledge Professor Paweł Krysiński for providing access to the ellipsometer.

\section{Author contributions}

W.L. gave the idea of the research. W.L., M.F., C.R. and E.G. designed the research. W.L. designed and produced the nanostructures. W.L. performed TEM, SAXRD and ultraviolet-visible measurements. M.F. and C.R. performed theoretical calculations. W.L. and E.G. performed and analysed ellipsometric measurements. W.L., M.F. and C.R. prepared the figures and W.L., M.F., J.M., C.R. and E.G. wrote the manuscript.

\section{Additional information}

Supplementary Information accompanies this paper at http://www.nature.com/ naturecommunications

Competing financial interests: The authors declare no competing financial interests.

Reprints and permission information is available online at http://npg.nature.com/ reprintsandpermissions/

How to cite this article: Lewandowski, W. et al. Dynamically self-assembled silver nanoparticles as a thermally tunable metamaterial. Nat. Commun. 6:6590 doi: 10.1038/ncomms7590 (2015) 